\title{
EXISTENCE RESULTS FOR VOLTERRA-STIELTJES QUADRATIC INTEGRAL EQUATIONS ON AN UNBOUNDED INTERVAL
}

\author{
JÓZEF BANAŚ
}

\begin{abstract}
We prove a few existence results for a nonlinear quadratic Volterra-Stieltjes integral equation on an unbounded interval. Our proof depends on suitable combination of the technique of measures of noncompactness and the Schauder fixed point principle. Such an approach permits us to obtain existence theorems under rather general assumptions.
\end{abstract}

\section{Introduction}

It is well-known that a lot of problems investigated in mechanics, engineering, mathematical physics, biology, vehicular traffic theory, queuing theory and also several real world problems can be described with help of various integral equations, both linear and nonlinear (cf. [1], [13], [15], [18], [25]). The theory of integral equations is highly developed and constitutes a significant and important branch of nonlinear analysis. There have been published, up to now, numerous research papers containing, among others, many existence results for integral equations of various types.

The aim of this paper is to investigate the solvability of a class of nonlinear quadratic Volterra-Stieltjes integral equations. In the case when we look for solutions of the mentioned integral equations which are defined on a bounded interval we can apply successfully the technique of measures of noncompactness.

That technique enables us to obtain existence results under rather general assumptions [4], [5], [8], [11]. But in the case when we look for solutions of Volterra-Stieltjes integral equations defined on an unbounded interval the situation is more complicated. Namely, the existence results for the class of integral equations of such a type were obtained under rather restrictive assumptions [9], [10]. Let us mention that existence results for even simpler type of integral equations on an unbounded interval were also proved under rather restrictive assumptions (cf. [13], [15], [18], [24], [25], for example). 
In this paper we prove some existence theorems for a class of VolterraStieltjes quadratic integral equations containing numerous types of Volterra integral equations as special cases. We investigate solvability of those integral equations in the space of bounded continuous functions defined on an unbounded interval. The main tool used in our considerations is the combination of the technique of measures of noncompactness with the Schauder fixed point principle. Such a proof enables us to obtain our existence results under quite general and convenient assumptions.

The results proved in this paper generalize several ones obtained up to now for various types of nonlinear Volterra integral equations.

\section{Auxiliary facts and results}

In this section we present some basic facts and results which will be used in further considerations. If $x$ is a real function defined on the interval $[a, b]$, then the symbol $\bigvee_{a}^{b} x$ denotes the variation of $x$ on $[a, b]$. We say that $x$ is of bounded variation on the interval $[a, b]$ whenever $\bigvee_{a}^{b} x$ is finite. If $h(t, s)=$ $h:[a, b] \times[c, b] \rightarrow \mathrm{R}$, then the symbol $\bigvee_{t=p}^{q} h(t, s)$ indicates the variation of the function $t \rightarrow h(t, s)$ on the interval $[p, q] \subset[a, b]$, where $s$ is arbitrarily fixed in $[c, d]$. In the same way we define $\bigvee_{s=p}^{q} h(t, s)$. For the properties of functions of bounded variation we refer to [19], [21].

If $x$ and $\varphi$ are two real functions defined on the interval $[a, b]$, then under some conditions (see [21]) we can define the Stieltjes integral (in the RiemannStieltjes sense)

$$
\int_{a}^{b} x(t) d \varphi(t)
$$

of the function $x$ with respect to $\varphi$. In this case we say that $x$ is Stieltjes integrable on $[a, b]$ with respect to $\varphi$. Several conditions are known guaranteeing Stieltjes integrability [21], [22]. One of the most frequently used requires that $x$ is continuous and $\varphi$ is of bounded variation on $[a, b]$.

In what follows we will use a few properties of the Stieltjes integral contained in the below given lemmas (cf. [20]).

Lemma 1. If $x$ is Stieltjes integrable on the interval $[a, b]$ with respect to a function $\varphi$ of bounded variation then

$$
\left|\int_{a}^{b} x(t) d \varphi(t)\right| \leq \int_{a}^{b}|x(t)| d\left(\bigvee_{a}^{t} \varphi\right)
$$

Lemma 2. Let $x_{1}, x_{2}$ be Stieltjes integrable functions on the interval $[a, b]$ with respect to a nondecreasing function $\varphi$ such that $x_{1}(t) \leq x_{2}(t)$ for $t \in$ 
$[a, b]$. Then

$$
\int_{a}^{b} x_{1}(t) d \varphi(t) \leq \int_{a}^{b} x_{2}(t) d \varphi(t)
$$

In the sequel we will also consider Stieltjes integrals of the form

$$
\int_{a}^{b} x(s) d_{s} g(t, s)
$$

where $g:[a, b] \times[a, b] \rightarrow \mathrm{R}$ and the symbol $d_{s}$ indicates the integration with respect to $s$. The details concerning this integral will be described later.

Now we assume that $x$ is a real function defined on $[a, b]$. Denote by $\omega(x, \varepsilon)$ the modulus of continuity of the function $x$, i.e.

$$
\omega(x, \varepsilon)=\sup \{|x(t)-x(s)|: t, s \in[a, b],|t-s| \leq \varepsilon\} .
$$

If $p(t, s)=p:[a, b] \times[c, d] \rightarrow \mathrm{R}$, we define the modulus of continuity of $p(t, s)$ with respect to each variable separately. For example

$$
\omega(p(t, \cdot), \varepsilon)=\sup \{|p(t, u)-p(t, v)|: u, v \in[c, d],[u-v] \leq \varepsilon\},
$$

where $t$ is a fixed number in the interval $[a, b]$.

Now we collect some facts concerning measures of noncompactness needed further on. Let $(E,\|\cdot\|)$ be an infinite dimensional Banach space with zero element $\theta$. Denote by $B(x, r)$ the closed ball centered at $x$ and with radius $r$. The symbol $B_{r}$ stands for the ball $B(\theta, r)$. For a subset $X$ of $E$ we write $\bar{X}$, Conv $X$ in order to denote the closure and convex closure of $X$, respectively. The family of all nonempty and bounded subsets of $E$ is denoted by $\mathfrak{M}_{E}$ and its subfamily consisting of all relatively compact sets is denoted by $\mathfrak{N}_{E}$.

We use the following definition of the concept of a measure of noncompactness [6].

Definition. A mapping $\mu: \mathfrak{M}_{E} \rightarrow \mathbf{R}_{+}=[0,+\infty)$ is said to be a measure of noncompactness in $E$ if it satisfies the following conditions:

$1^{o}$ The family ker $\mu=\left\{X \in \mathfrak{M}_{E}: \mu(X)=0\right\}$ is nonempty and ker $\mu \subset$ $\Re_{E}$

$$
\begin{aligned}
& 2^{o} \quad X \subset Y \Rightarrow \mu(X) \leq \mu(Y) \\
& 3^{o} \mu(\bar{X})=\mu(X) \\
& 4^{o} \mu(\operatorname{Conv} X)=\mu(X) \\
& 5^{o} \mu(\lambda X+(1-\lambda) Y) \leq \lambda \mu(X)+(1-\lambda) \mu(Y) \text { for } \lambda \in[0,1]
\end{aligned}
$$


$6^{o}$ If $\left(X_{n}\right)$ is a sequence of closed sets from $\mathfrak{M}_{E}$ such that $X_{n+1} \subset X_{n}$ $(n=1,2, \ldots)$ and if $\lim _{n \rightarrow \infty} \mu\left(X_{n}\right)=0$ then the intersection $X_{\infty}=$ $\bigcap_{n=1}^{\infty} X_{n}$ is nonempty.

The family $\operatorname{ker} \mu$ described in $1^{o}$ is called the kernel of the measure of noncompactness $\mu$. Let us observe that the intersection set $X_{\infty}$ from $6^{o}$ belongs to ker $\mu$. Indeed, $\mu\left(X_{\infty}\right) \leq \mu\left(X_{n}\right)$ for every $n$ which implies that $\mu\left(X_{\infty}\right)=0$. This simple observation will be crucial in our further considerations. Further facts concerning measures of noncompactness and its properties may be found in [6].

In what follows we will work in the Banach space $B C\left(\mathrm{R}_{+}\right)$consisting of all real functions defined, bounded and continuous on $\mathbf{R}_{+}$. The space $B C\left(\mathbf{R}_{+}\right)$ is furnished with the standard norm

$$
\|x\|=\sup \{|x(t)|: t \geq 0\} .
$$

Let us describe the measure of noncompactness which will be used in further investigations (cf. [6]). Let us fix a nonempty bounded subset $X$ of $B C\left(\mathrm{R}_{+}\right)$ and a positive number $T>0$. For $x \in X$ and $\varepsilon>0$ denote by $\omega^{T}(x, \varepsilon)$ the modulus of continuity of $x$ on the interval $[0, T]$. Further, let us put:

$$
\begin{aligned}
\omega^{T}(X, \varepsilon) & =\sup \left\{\omega^{T}(x, \varepsilon): x \in X\right\}, \\
\omega_{0}^{T}(X) & =\lim _{\varepsilon \rightarrow 0} \omega^{T}(X, \varepsilon), \\
\omega_{0}(X) & =\lim _{T \rightarrow \infty} \omega_{0}^{T}(X) .
\end{aligned}
$$

Moreover, let us put:

$$
\beta(X)=\lim _{T \rightarrow \infty}\left\{\sup _{x \in X}\{\sup [|x(t)|: t \geq T]\}\right\} .
$$

Finally, we define the function $\mu$ on the family $\mathfrak{M}_{B C\left(\mathrm{R}_{+}\right)}$in the following way

$$
\mu(X)=\omega_{0}(X)+\beta(X) .
$$

It can be shown [6] that the function $\mu$ is a measure of noncompactness in the space $B C\left(\mathrm{R}_{+}\right)$. The kernel ker $\mu$ of this measure contains nonempty and bounded sets $X$ such that functions from $X$ are locally equicontinuous on $\mathbf{R}_{+}$ and tend to zero at infinity uniformly with respect to the set $X$, i.e. for any $\varepsilon>0$ there exists $T>0$ such that $|x(t)|<\varepsilon$ for all $t \geq T$ and $x \in X$. 


\section{Main results}

We are going to study the nonlinear quadratic integral equation of VolterraStieltjes type having the form

$$
x(t)=a(t)+f(t, x(t)) \int_{0}^{t} u(t, \tau, x(\tau)) d_{\tau} g(t, \tau), \quad t \geq 0 .
$$

We look for solutions of this equation in the space $B C\left(\mathrm{R}_{+}\right)$. In our investigations we assume that the following conditions are satisfied:

(i) $a: \mathbf{R}_{+} \rightarrow \mathbf{R}$ is a continuous function such that $a(t) \rightarrow 0$ as $t \rightarrow \infty$;

(ii) $g: \mathbf{R}_{+} \times \mathbf{R}_{+} \rightarrow \mathbf{R}$ and for all $t_{1}, t_{2} \in \mathbf{R}_{+}$such that $t_{1}<t_{2}$ the function $s \rightarrow g\left(t_{2}, s\right)-g\left(t_{1}, s\right)$ is nondecreasing on $\mathbf{R}_{+}$;

(iii) the function $s \rightarrow g(0, s)$ is nondecreasing on $\mathbf{R}_{+}$;

(iv) the functions $s \rightarrow g(t, s)$ and $t \rightarrow g(t, s)$ are continuous on $\mathbf{R}_{+}$for each fixed $t \in \mathbf{R}_{+}$or $s \in \mathbf{R}_{+}$, respectively;

(v) the function $u: \mathbf{R}_{+} \times \mathbf{R}_{+} \times \mathbf{R} \rightarrow \mathbf{R}$ is continuous and there exist a continuous function $k(t, s)=k: \mathbf{R}_{+} \times \mathbf{R}_{+} \rightarrow \mathbf{R}_{+}$and a continuous and nondecreasing function $\varphi: \mathbf{R}_{+} \rightarrow \mathbf{R}_{+}$such that

$$
|u(t, s, x)| \leq k(t, s) \varphi(|x|)
$$

for all $t, s \in \mathrm{R}_{+}$and $x \in \mathrm{R}$;

(vi) $f: \mathbf{R}_{+} \times \mathbf{R} \rightarrow \mathbf{R}$ is a continuous function and there exists a continuous function $m: \mathbf{R}_{+} \rightarrow \mathbf{R}_{+}$such that

$$
|f(t, x)-f(t, y)| \leq m(t)|x-y|
$$

for all $x, y \in \mathbf{R}$ and $t \in \mathbf{R}_{+}$. Moreover, $f(t, 0) \rightarrow 0$ as $t \rightarrow \infty$;

(vii) the functions $t \rightarrow \int_{0}^{t} k(t, s) d_{s} g(t, s)$ and $t \rightarrow m(t) \int_{0}^{t} k(t, s) d_{s} g(t, s)$ are bounded on $\mathbf{R}_{+}$.

For further purposes let us define the following constants:

$$
\begin{aligned}
& F=\sup \{|f(t, 0)|: t \geq 0\}, \\
& K=\sup \left\{\int_{0}^{t} k(t, s) d_{s} g(t, s): t \geq 0\right\}, \\
& M=\sup \left\{m(t) \int_{0}^{t} k(t, s) d_{s} g(t, s): t \geq 0\right\} .
\end{aligned}
$$

Obviously all these constants are finite in view of assumptions (vi) and (vii). 
Now we state a few simple consequences of the above conditions.

Lemma 3. Under assumptions (ii) and (iii) the function $s \rightarrow g(t, s)$ is nondecreasing on $\mathbf{R}_{+}$for any fixed $t \in \mathbf{R}_{+}$.

Proof. Take arbitrary $s_{1}, s_{2} \in \mathbf{R}_{+}$with $s_{1}<s_{2}$. Then, in view of (iii) we have

$$
g\left(0, s_{2}\right)-g\left(0, s_{1}\right) \geq 0 .
$$

Hence we get

$$
\begin{aligned}
g\left(t, s_{2}\right)-g\left(t, s_{1}\right) & \geq\left[g\left(t, s_{2}\right)-g\left(t, s_{1}\right)\right]-\left[g\left(0, s_{2}\right)-g\left(0, s_{1}\right)\right] \\
& =\left[g\left(t, s_{2}\right)-g\left(0, s_{2}\right)\right]-\left[g\left(t, s_{1}\right)-g\left(0, s_{1}\right)\right] .
\end{aligned}
$$

But the last obtained expression is nonnegative in view of assumption (ii). This completes the proof.

Remark 1. From the above lemma we infer that the function $s \rightarrow g(t, s)$ is of bounded variation on each bounded interval $[a, b] \subset \mathbf{R}_{+}$, for any fixed $t \in \mathbf{R}_{+}$. This fact is used in our considerations. For example, it implies that the integrals in equation (1) and in assumption (vii) are well-defined.

Lemma 4. Assume that the function $g$ satisfies assumption (ii). Then, for arbitrarily fixed $s_{1}, s_{2} \in \mathrm{R}_{+}$such that $s_{1}<s_{2}$, the function $t \rightarrow g\left(t, s_{2}\right)-$ $g\left(t, s_{1}\right)$ is nondecreasing on $\mathbf{R}_{+}$.

A simple proof can be found in [4].

Next, let us assume the following additional assumption:

(viii) there exists a positive solution $r_{0}$ of the inequality

$$
\|a\|+F K \varphi(r)+\operatorname{Mr} \varphi(r) \leq r
$$

such that $M \varphi\left(r_{0}\right)<1$.

Remark 2. Observe that if $r_{0}$ satisfies inequality (2) and $r_{0}>0$ then

$$
M \varphi\left(r_{0}\right) \leq 1-\frac{\|a\|}{r_{0}}-\frac{F K \varphi\left(r_{0}\right)}{r_{0}} .
$$

Thus, in the case when $F K \neq 0$ or the function $a=a(t)$ does not vanish on $\mathrm{R}_{+}$, the condition $M \varphi\left(r_{0}\right)<1$ is automatically satisfied.

Now we can formulate our main existence result.

THEOREM 1. Under assumptions (i)-(viii) equation (1) has at least one solution $x=x(t)$ belonging to the space $B C\left(\mathrm{R}_{+}\right)$such that $x(t) \rightarrow 0$ as $t \rightarrow \infty$. 
Proof. Consider the operator $U$ defined on the space $B C\left(\mathrm{R}_{+}\right)$by the formula

$$
(U x)(t)=a(t)+f(t, x(t)) \int_{0}^{t} u(t, \tau, x(\tau)) d_{\tau} g(t, \tau) .
$$

Taking into account assumptions (i), (ii), (iii), (v) and Lemma 3 (cf. also Remark 1) we deduce that $U$ is well-defined on the space $B C\left(\mathbf{R}_{+}\right)$.

Further, fix arbitrarily $T>0$ and $\varepsilon>0$. Take $t, s \in[0, T]$ such that $s<t$ and $t-s \leq \varepsilon$. Then, in view of our assumptions and Lemmas 1 and 2, for an arbitrary function $x \in B C\left(\mathrm{R}_{+}\right)$we obtain:

$$
\begin{aligned}
& \text { (3) }|(U x)(t)-(U x)(s)| \\
& \leq \omega^{T}(a, \varepsilon)+|f(t, x(t))-f(t, x(s))| \cdot\left|\int_{0}^{t} u(t, \tau, x(\tau)) d_{\tau} g(t, \tau)\right| \\
& +\left|f(t, x(s)) \int_{0}^{t} u(t, \tau, x(\tau)) d_{\tau} g(t, \tau)-f(s, x(s)) \int_{0}^{t} u(t, \tau, x(\tau)) d_{\tau} g(t, \tau)\right| \\
& +\left|f(s, x(s)) \int_{0}^{t} u(t, \tau, x(\tau)) d_{\tau} g(t, \tau)-f(s, x(s)) \int_{0}^{t} u(t, \tau, x(\tau)) d_{\tau} g(s, \tau)\right| \\
& +\left|f(s, x(s)) \int_{0}^{t} u(t, \tau, x(\tau)) d_{\tau} g(s, \tau)-f(s, x(s)) \int_{0}^{t} u(s, \tau, x(\tau)) d_{\tau} g(s, \tau)\right| \\
& +\left|f(s, x(s)) \int_{0}^{t} u(s, \tau, x(\tau)) d_{\tau} g(s, \tau)-f(s, x(s)) \int_{0}^{s} u(s, \tau, x(\tau)) d_{\tau} g(s, \tau)\right| \\
& \leq \omega^{T}(a, \varepsilon)+m(t)|x(t)-x(s)| \int_{0}^{t}|u(t, \tau, x(\tau))| d_{\tau}\left(\bigvee_{p=0}^{\tau} g(t, p)\right) \\
& +|f(t, x(s))-f(s, x(s))| \int_{0}^{t}|u(t, \tau, x(\tau))| d_{\tau}\left(\bigvee_{p=0}^{\tau} g(t, p)\right) \\
& +|f(s, x(s))| \int_{0}^{t}|u(t, \tau, x(\tau))| d_{\tau}\left(\bigvee_{p=0}^{\tau}[g(t, p)-g(s, p)]\right) \\
& +|f(s, x(s))| \int_{0}^{t}|u(t, \tau, x(\tau))-u(s, \tau, x(\tau))| d_{\tau}\left(\bigvee_{p=0}^{\tau} g(s, p)\right) \\
& +|f(s, x(s))| \int_{s}^{t}|u(s, \tau, x(\tau))| d_{\tau}\left(\bigvee_{p=s}^{\tau} g(s, p)\right) \\
& \leq \omega^{T}(a, \varepsilon)+m(t) \omega^{T}(x, \varepsilon) \int_{0}^{t} k(t, \tau) \varphi(|x(\tau)|) d_{\tau} g(t, \tau) \\
& +
\end{aligned}
$$




$$
\begin{aligned}
& +\omega_{f}^{T}(\varepsilon) \int_{0}^{t} k(t, \tau) \varphi(|x(\tau)|) d_{\tau} g(t, \tau) \\
& +[m(s)|x(s)|+|f(s, 0)|] \int_{0}^{t} k(t, \tau) \varphi(|x(\tau)|) d_{\tau}(g(t, \tau)-g(s, \tau)) \\
& +[m(s)|x(s)|+|f(s, 0)|] \int_{0}^{t} \omega_{u}^{T}(\varepsilon) d_{\tau} g(s, \tau) \\
& +[m(s)|x(s)|+|f(s, 0)|] \int_{s}^{t} k(s, \tau) \varphi(|x(\tau)|) d_{\tau} g(s, \tau),
\end{aligned}
$$

where

$$
\begin{aligned}
& \omega_{f}^{T}(\varepsilon)=\sup \{|f(t, y)-f(s, y)|: \\
& \qquad, s \in[0, T],|t-s| \leq \varepsilon, y \in[-\|x\|,\|x\|]\}, \\
& \omega_{u}^{T}(\varepsilon)=\sup \{|u(t, \tau, y)-u(s, \tau, y)|: \\
& t, s, \tau \in[0, T],|t-s| \leq \varepsilon, y \in[-\|x\|,\|x\|]\} .
\end{aligned}
$$

For our further purposes, let us put

$$
\begin{aligned}
k_{T} & =\sup \{k(t, s): t, s \in[0, T]\}, \\
f_{T} & =\sup \{|f(t, 0)|: t \in[0, T]\}, \\
m_{T} & =\sup \{m(t): t \in[0, T]\} .
\end{aligned}
$$

Then, form estimate (3) we get

$$
\begin{aligned}
& |(U x)(t)-(U x)(s)| \\
& \leq \omega^{T}(a, \varepsilon)+M \varphi(\|x\|) \omega^{T}(x, \varepsilon)+K \varphi(\|x\|) \omega_{f}^{T}(\varepsilon) \\
& \quad+\left(m_{T}\|x\|+f_{T}\right)\left\{k_{T} \varphi(\|x\|) \int_{0}^{t} d_{\tau}(g(t, \tau)-g(s, \tau))\right. \\
& \left.\quad+\omega_{u}^{T}(\varepsilon) \int_{0}^{t} d_{\tau} g(s, \tau)+k_{T} \varphi(\|x\|) \int_{s}^{t} d_{\tau} g(s, \tau)\right\} \\
& =\omega^{T}(a, \varepsilon)+M \varphi(\|x\|) \omega^{T}(x, \varepsilon)+K \varphi(\|x\|) \omega_{f}^{T}(\varepsilon) \\
& \quad+\left(m_{T}\|x\|+f_{\tau}\right)\left\{k_{T} \varphi(\|x\|)([g(t, t)-g(s, t)]-[g(t, 0)-g(s, 0)])\right. \\
& \left.\quad+\omega_{u}^{T}(\varepsilon)[g(s, t)-g(s, 0)]+k_{T} \varphi(\|x\|)[g(s, t)-g(s, s)]\right\} .
\end{aligned}
$$


Next, keeping in mind Lemmas 3 and 4, from the above estimate we derive at

(4) $|(U x)(t)-(U x)(s)|$

$$
\begin{aligned}
& \leq \omega^{T}(a, \varepsilon)+M \varphi(\|x\|) \omega^{T}(x, \varepsilon)+K \varphi(\|x\|) \omega_{f}^{T}(\varepsilon) \\
& \quad+\left(m_{T}\|x\|+f_{T}\right)\left\{k_{T} \varphi(\|x\|) \cdot([g(t, T)-g(s, T)]\right. \\
& \quad+|g(t, 0)-g(s, 0)|)+\omega_{u}^{T}(\varepsilon)[g(T, t)-g(T, 0)] \\
& \left.\quad+k_{T} \varphi(\|x\|)[g(T, t)-g(T, s)]\right\} \\
& \leq \omega^{T}(a, \varepsilon)+M \varphi(\|x\|) \omega^{T}(x, \varepsilon)+K \varphi(\|x\|) \omega_{f}^{T}(\varepsilon) \\
& \quad+\left(m_{T}\|x\|+f_{T}\right)\left\{k_{T} \varphi(\|x\|)\left[\omega^{T}(g(\cdot, T), \varepsilon)+\omega^{T}(g(\cdot, 0), \varepsilon)\right]\right. \\
& \left.\quad+\omega_{u}^{T}(\varepsilon)[g(T, T)-g(T, 0)]+k_{T} \varphi(\|x\|) \omega^{T}(g(T, \cdot), \varepsilon)\right\} .
\end{aligned}
$$

Now, let us observe that from the uniform continuity of the function $f(t, y)$ on the set $[0, T] \times[-\|x\|,\|x\|]$ and the function $u(t, \tau, y)$ on the set $[0, T] \times$ $[0, T] \times[-\|x\|,\|x\|]$ we infer that $\omega_{f}^{T}(\varepsilon) \rightarrow 0$ and $\omega_{u}^{T}(\varepsilon) \rightarrow 0$ as $\varepsilon \rightarrow 0$. Thus, keeping in mind these facts, the assumption (iv) and the estimate (4) we deduce that the function $U x$ is continuous on the interval $[0, T]$. In view of the arbitrariness of $T$ we conclude that $U x$ is continuous on the whole interval $\mathrm{R}_{+}$.

In order to show that $U x$ is bounded on $\mathbf{R}_{+}$let us fix arbitrarily $t \in \mathbf{R}_{+}$. Then, using our assumptions and Lemmas 1 and 2 we get

(5) $|(U x)(t)|$

$$
\begin{aligned}
& \leq|a(t)|+|f(t, x(t))| \int_{0}^{t}|u(t, \tau, x(\tau))| d_{\tau}\left(\bigvee_{p=0}^{\tau} g(t, p)\right) \\
& \leq|a(t)|+[m(t)|x(t)|+|f(t, 0)|] \int_{0}^{t} k(t, \tau) \varphi(|x(\tau)|) d_{\tau} g(t, \tau) \\
& \leq|a(t)|+|f(t, 0)| \varphi(\|x\|) \int_{0}^{t} k(t, \tau) d_{\tau} g(t, \tau) \\
& \quad+\varphi(\|x\|)|x(t)| m(t) \int_{0}^{t} k(t, \tau) d_{\tau} g(t, \tau) \\
& \leq|a(t)|+|f(t, 0)| \varphi(\|x\|) \int_{0}^{t} k(t, \tau) d_{\tau} g(t, \tau)+|x(t)| M \varphi(\|x\|) .
\end{aligned}
$$

Hence we get

$$
|(U x)(t)| \leq\|a\|+F K \varphi(\|x\|)+M\|x\| \varphi(\|x\|),
$$


where $F$ and $K$ are constants defined earlier.

From the above estimate we infer that $U x$ is bounded on $\mathrm{R}_{+}$. This fact, in conjunction with the continuity of the function $U x$, allows us to deduce that the operator $U$ transforms the space $B C\left(\mathbf{R}_{+}\right)$into itself.

In what follows let us observe that inequality (6) yields

$$
\|U x\| \leq\|a\|+F K \varphi(\|x\|)+M\|x\| \varphi(\|x\|) .
$$

Combining this estimate with assumption (viii) we conclude that there exists a positive number $r_{0}$ such that $M \varphi\left(r_{0}\right)<1$ and that the operator $U$ transforms the ball $B_{r_{0}}$ into itself.

Now, let us take a nonempty subset $X$ of the ball $B_{r_{0}}$. Fix arbitrarily $T>0$ and $\varepsilon>0$. Then, using the previously obtained estimate (4) (where in the definition of the functions $\omega_{f}^{T}(\varepsilon)$ and $\omega_{u}^{T}(\varepsilon)$ the quantity $\|x\|$ is replaced by $r_{0}$ ), for an arbitrary $x \in X$ we derive at

$$
\begin{aligned}
\omega^{T}(U x, \varepsilon) \leq \omega^{T}(a, \varepsilon)+M \varphi\left(r_{0}\right) \omega^{T}(x, \varepsilon)+K \varphi\left(r_{0}\right) \omega_{f}^{T}(\varepsilon) \\
+\left(m_{T} r_{0}+f_{T}\right)\left\{k_{T} \varphi\left(r_{0}\right)\left[\omega^{T}(g(\cdot, T), \varepsilon)+\omega^{T}(g(\cdot, 0), \varepsilon)\right]\right. \\
\left.+\omega_{u}^{T}(\varepsilon)[g(T, T)-g(T, 0)]+k_{T} \varphi\left(r_{0}\right) \omega^{T}(g(T, \cdot), \varepsilon)\right\} .
\end{aligned}
$$

Hence, keeping in mind our assumptions and the facts established before, we obtain

$$
\omega_{0}(U X) \leq M \varphi\left(r_{0}\right) \omega_{0}(X) .
$$

Next, fix $T>0$ and take an arbitrary function $x \in X$. Then, using estimate (5) for $t \geq T$, we get

$$
|(U x)(t)| \leq|a(t)|+|f(t, 0)| K \varphi\left(r_{0}\right)+M \varphi\left(r_{0}\right)|x(t)| .
$$

This estimate, in conjunction with assumptions (i) and (vi), yields

$$
\beta(U X) \leq M \varphi\left(r_{0}\right) \beta(X),
$$

where the function $\beta=\beta(X)$ was defined in Section 2 .

Finally, let us observe that linking (7) and (8) we have

$$
\mu(U X) \leq M \varphi\left(r_{0}\right) \mu(X),
$$

where $\mu$ is the measure of noncompactness defined in the previous section.

In what follows let us put $B_{r_{0}}^{1}=\operatorname{Conv} U\left(B_{r_{0}}\right), B_{r_{0}}^{2}=\operatorname{Conv} U\left(B_{r_{0}}^{1}\right)$ and so on. Observe that the sequence of sets $\left(B_{r_{0}}^{n}\right)$ is decreasing i.e. $B_{r_{0}}^{n+1} \subset B_{r_{0}}^{n}$ for 
$n=1,2, \ldots$ Moreover, the sets of this sequence are closed and convex. From this, in view of (9), we get

$$
\mu\left(B_{r_{0}}^{n}\right) \leq q^{n} \mu\left(B_{r_{0}}\right),
$$

where we put $q=M \varphi\left(r_{0}\right)$. Notice that $q<1$, in view of assumption (viii). Moreover, we can easily calculate that $\mu\left(B_{r_{0}}\right)=3 r_{0}$. Combining this fact and (10) we obtain

$$
\lim _{n \rightarrow \infty} \mu\left(B_{r_{0}}^{n}\right)=0 .
$$

Hence, taking into account axiom $6^{\circ}$ of the definition of a measure of noncompactness, we infer that the set $Y=\bigcap_{n=1}^{\infty} B_{r_{0}}^{n}$ is nonempty, bounded, closed and convex. Moreover, the set $Y$ is a member of the kernel ker $\mu$ of the measure of noncompactness $\mu$ (cf. remark made after the mentioned definition). We observe that the operator $U$ transforms the set $Y$ into itself.

In the sequel we show that $U$ is continuous on the set $Y$. To do this let us fix a number $\varepsilon>0$ and take arbitrary functions $x, y \in Y$ such that $\|x-y\| \leq \varepsilon$. Using the fact that $Y \in \operatorname{ker} \mu$ and the description of sets belonging to $\operatorname{ker} \mu$ given in Section 2, we find a number $T>0$ such that for any function $z \in Y$ and for $t \geq T$ the inequality $|z(t)| \leq \varepsilon$ is true. Moreover, keeping in mind assumption (vi), we can choose $T$ in such a way that $|f(t, 0)| \leq \varepsilon$ for $t \geq T$. Then, using our assumptions and Lemmas 1 and 2 , for an arbitrary $t \geq T$ we get

$$
\begin{aligned}
& |(U x)(t)-(U y)(t)| \\
& \leq \varepsilon m(t) \int_{0}^{t} k(t, \tau) \varphi(|x(\tau)|) d_{\tau} g(t, \tau)+[m(t)|y(t)|+|f(t, 0)|] \\
& \quad \int_{0}^{t}[|u(t, \tau, x(\tau))|+|u(t, \tau, y(\tau))|] d_{\tau}\left(\bigvee_{p=0}^{\tau} g(t, p)\right) \\
& \leq \varepsilon \varphi\left(r_{0}\right) M+2 \varepsilon \varphi\left(r_{0}\right) m(t) \int_{0}^{t} k(t, \tau) d_{\tau} g(t, \tau) \\
& +2 \varepsilon \varphi\left(r_{0}\right) \int_{0}^{t} k(t, \tau) d_{\tau} g(t, \tau) \leq \varepsilon \varphi\left(r_{0}\right)(3 M+2 k)
\end{aligned}
$$

Further, let us assume that $t \in[0, T]$. Then, applying the imposed assumptions and evaluating similary as above, we obatin

$$
\begin{aligned}
& |(U x)(t)-(U y)(t)| \\
& \quad \leq \varepsilon m(t) \int_{0}^{t} k(t, \tau) \varphi(|x(\tau)|) d_{\tau} g(t, \tau)
\end{aligned}
$$




$$
\begin{aligned}
&+[m(t)|y(t)|+|f(t, 0)|] \int_{0}^{t}|u(t, \tau, x(\tau))-u(t, \tau, y(\tau))| d_{\tau} g(t, \tau) \\
& \leq \varepsilon \varphi\left(r_{0}\right) M+\left(r_{0} m_{T}+f_{T}\right) \bar{\omega}_{u}^{T}(\varepsilon)[g(t, t)-g(t, 0)],
\end{aligned}
$$

where

$\bar{\omega}_{u}^{T}(\varepsilon)$

$=\sup \left\{|u(t, \tau, x)-u(t, \tau, y)|: t, \tau \in[0, T], x, y \in\left[-r_{0}, r_{0}\right],|x-y| \leq \varepsilon\right\}$.

Hence, applying Lemmas 3 and 4, we derive at the following estimate:

$$
\begin{aligned}
\mid(U x)(t)- & (U y)(t) \mid \\
& \leq \varepsilon M \varphi\left(r_{0}\right)+\left(r_{0} m_{T}+f_{T}\right) \bar{\omega}_{u}^{T}(\varepsilon)[g(T, T)-g(T, 0)] .
\end{aligned}
$$

Observe that in view of the uniform continuity of the function $u=u(t, \tau, x)$ on the set $[0, T] \times[0, T] \times\left[-r_{0}, r_{0}\right]$ we deduce that $\bar{\omega}_{u}^{T}(\varepsilon) \rightarrow 0$ as $\varepsilon \rightarrow 0$. Combining this fact, (11) and (12), we infer that the operator $U$ is continuous on the set $Y$.

Finally, taking into account all facts concerning the set $Y$ and the operator $U: Y \rightarrow Y$ which were established above, in view of the Schauder fixed point principle, we conclude that $U$ has at least one fixed point $x$ in the set $Y$. Obviously the function $x=x(t)$ is a solution of the equation (1). Moreover, keeping in mind the fact that $Y \in \operatorname{ker} \mu$, we infer that $x(t) \rightarrow 0$ as $t \rightarrow \infty$. This completes the proof.

REMARK 3. Let us mention that all fixed points of the operator $U$ belonging to the ball $B_{r_{0}}$ belong also to the set $Y$. This fact is a simple consequence of the construction of $Y$. Thus, all solutions $x=x(t)$ of equation (1) belonging to the ball $B_{r_{0}}$ satisfy the condition $x(t) \rightarrow 0$ as $t \rightarrow \infty$.

It can be shown that Theorem 1 covers many special cases of equation (1). Nevertheless, there are some interesting and important cases of equation (1) which are not covered by Theorem 1 . Indeed, in order to obtain a result on the existence of solutions $x=x(t)$ of the equation (1) vanishing at infinity (i.e. such that $x(t) \rightarrow 0$ as $t \rightarrow \infty)$, we required in Theorem 1 that $f(t, 0) \rightarrow 0$ as $t \rightarrow \infty$. It turns out that for some important cases of equation (1) this condition is not satisfied. For example, when $f(t, x) \equiv 1$ or $f(t, x)=h(t)$ and $h(t)$ does not vanish at infinity then Theorem 1 fails to work.

Below we formulate an existence result concerning the above mentioned cases of equation (1).

THEOREM 2. Assume that the assumptions of Theorem 1 are satisfied except assumption (vi) where, instead of the condition that $f(t, 0) \rightarrow 0$ as $t \rightarrow \infty$, 
we require that the function $t \rightarrow f(t, 0)$ belongs to the space $B C\left(\mathrm{R}_{+}\right)$, and in assumption (viii) where we require additionally that

$$
\lim _{t \rightarrow \infty} \int_{0}^{t} k(t, s) d_{s} g(t, s)=0 .
$$

Then equation (1) has at least one solution $x=x(t)$ such that $x \in B C\left(\mathbf{R}_{+}\right)$ and $x(t)$ vanishes at infinity.

The proof of the above theorem is similar to the proof of Theorem 1 and is therefore omitted.

\section{Further discussions, remarks and examples}

This section is devoted to discuss some details appearing in our existence results proved in the previous section. At the beginning we consider a few special cases of the equation (1).

First of all let us notice that the classical nonlinear Volterra integral equation

$$
x(t)=a(t)+\int_{0}^{t} u(t, s, x(s)) d s
$$

is a special case of equation (1) if we put $f(t, x) \equiv 1$ and $g(t, s)=s$. This implies that equation (13) can be investigated with help of Theorem 2. Observe that some assumptions of that theorem are automatically satisfied for equation (13). Indeed, assumptions (ii), (iii) and (iv) are trivially satisfied. Assumption (vi) is satisfied with $m(t) \equiv 0$ and $f(t, 0) \equiv 1$. Thus we have to assume hypotheses (i), (v) and the following ones,

(vii') the function $t \rightarrow \int_{0}^{t} k(t, s) d s$ is bounded on $\mathbf{R}_{+}$and tends to zero at infinity;

(viii') there exists a positive solution $r_{0}$ of the inequality $\|a\|+k \varphi(r) \leq r$.

Let us notice that in the case of equation (13) we have $F=1$ and $M=0$, where $F$ and $M$ are the constants defined in Section 3.

Next, let us consider the quadratic integral equation of Volterra type having the form

$$
x(t)=a(t)+x(t) \int_{0}^{t} \frac{t}{t+s} u(t, s, x(s)) d s .
$$

This equation represents the Volterra counterpart of the famous Chandrasekhar quadratic integral equation which has numerous applications (cf. [2], [3], [7], [14], [17]). In the case of a bounded interval this equation was discussed in [5], for example. 
We now show that equation (14) is a special case of equation (1). To do this let us consider the function $g: \mathbf{R}_{+} \times \mathbf{R}_{+} \rightarrow \mathbf{R}$ defined by the formula

$$
g(t, s)= \begin{cases}t \ln \frac{t+s}{t} & \text { for } t>0 \text { and } s \geq 0 \\ 0 & \text { for } t=0 \text { and } s \geq 0 .\end{cases}
$$

Then we see that equation (14) can be written in the form (1).

In order to discuss the existence result for equation (14) let us first notice that $f(t, x)=x$. This suggests that the existence result concerning equation (14) is contained in Theorem 1, where we have to assume hypotheses (i) and (v). Using standard methods of mathematical analysis it can be seen that the function $g(t, s)$ defined by (15) satisfies assumptions (ii), (iii) and (iv). Moreover, observe that assumption (vi) is satisfied with $m(t) \equiv 1$ and $f(t, 0) \equiv 0$ while assumptions (vii) and (viii) have the following form,

(vii") the function $t \rightarrow \int_{0}^{t} \frac{t k(t, s)}{t+s} d s$ is bounded on $\mathrm{R}_{+}$;

(viii") there exists a positive solution $r_{0}$ of the inequality $\|a\|+K r \varphi(r) \leq r$.

It is worthwhile mentioning that equation (1) contains also other special cases which are important in applications. For example, the following integral equation

$$
x(t)=f(t, x(t)) \int_{0}^{t} u(t, s, x(s)) d s
$$

plays a significant role in describing some problems connected with traffic theory and biology (see [18]). Obviously this equation is a special case of equation (1) if we put $a(t) \equiv 0$ and $g(t, s)=s$.

Now, we look at some concrete examples of the function $g=g(t, s)$ which appears in equation (1) and satisfies assumptions (ii), (iii) and (iv). Obviously the functions $g(t, s)=s$ and the function $g(t, s)$ defined by (15) are important examples of the functions in question. Moreover, the following functions

$$
\begin{aligned}
& g(t, s)=t s, \\
& g(t, s)=\min \{t, s\}, \\
& g(t, s)=\max \{t+s-1,0\}
\end{aligned}
$$

are examples of the function $g(t, s)$ satisfying our requirements. Let us mention that these functions are also examples of the so-called copulas playing an important role in some considerations in probability theory (cf. [16], [23], for instance).

Further, let us recall that if the function $g(t, s)$ satisfies assumptions (ii) and (iv) then it represents the distribution function of a two dimensional random 
variable [12], [22]. The converse implication is also "almost" valid [18], [22]. Particularly, we may consider the function as being the distribution function of a two dimensional random variable of continuous type. Such a function has the form

$$
g(t, s)=\int_{0}^{t}\left(\int_{0}^{s} p(z, y) d y\right) d z
$$

where $p(z, y)$ is the so-called density function [12]. Obviously this function satisfies assumptions (ii), (iii) and (iv). Moreover, we have

$$
d_{s} g(t, s)=\left(\int_{0}^{t} p(s, y) d y\right) d s
$$

and in this case equation (1) has the form

$$
x(t)=a(t)+f(t, x(t)) \int_{0}^{t} u(t, s, x(s))\left(\int_{0}^{t} p(s, y) d y\right) d s .
$$

Let us mention that this equation is the usual quadratic nonlinear Volterra integral equation.

Finally we provide two examples of concrete nonlinear quadratic integral equations of the form (1) which satisfy the assumptions formulated in Theorem 1 or 2 .

EXAMPLE 1. Consider the following quadratic integral equation of Volterra type

$$
x(t)=t \exp \left(-4 t^{2}\right)+(\exp (-t)+x(t)) \int_{0}^{t} \frac{x^{2}(s)}{1+t^{2}+s^{2}} d s .
$$

Obviously this equation is a special case of (1). Indeed, if we put

$$
\begin{aligned}
a(t) & =t \exp \left(-4 t^{2}\right), \\
f(t, x) & =\exp (-t)+x, \\
u(t, s, x) & =\frac{x^{2}}{1+t^{2}+s^{2}}, \\
g(t, s) & =s,
\end{aligned}
$$

then we can check that the assumptions of Theorem 1 are satisfied. In fact, we have $f(t, 0)=\exp (-t)$ which implies $F=1$. Further, we have $m(t) \equiv 1$ and

$$
|u(t, s, x)|=\frac{1}{1+t^{2}+s^{2}} x^{2},
$$


so we may put $k(t, s)=\frac{1}{1+t^{2}+s^{2}}$ and $\varphi(x)=x^{2}$. Hence we obtain

$$
\begin{aligned}
m(t) \int_{0}^{t} k(t, s) d_{s} g(t, s) & =\int_{0}^{t} k(t, s) d_{s} g(t, s) \\
& =\int_{0}^{t} \frac{1}{1+t^{2}+s^{2}} d s \\
& =\frac{1}{\sqrt{1+t^{2}}} \operatorname{arctg} \frac{t}{\sqrt{1+t^{2}}} .
\end{aligned}
$$

Consequently, we obtain at the following estimate:

$$
M=K \leq \pi / 4 .
$$

Moreover, we can easy calculate that $\|a\|=1 / \sqrt{8 e}$.

Finally, let us observe that the inequality from assumption (viii) has the form:

$$
1 / \sqrt{8 e}+K\left(r^{2}+r^{3}\right) \leq r .
$$

It is easily seen that this inequality has a positive solution $r_{0}$. For example, $r_{0}=2 / 5$.

EXAMPLE 2. Here we investigate the following quadratic equation of Volterra type:

(16) $x(t)=t^{2} \exp (-t)+(\operatorname{arctg} t+t x(t)) \int_{0}^{t} \frac{t}{t+s} \exp (-t-s) \sqrt{|x(s)|} d s$.

Observe that this equation has the form of equation (1) with the function $g(t, s)$ given by (15). This implies that assumptions (ii), (iii) and (iv) are satisfied. Further, let us notice that the terms involved in equation (1) have now the form

$$
\begin{aligned}
a(t) & =t^{2} \exp (-t), \\
f(t, x) & =\operatorname{arctg} t+t x, \\
u(t, s, x) & =\exp (-t-s) \sqrt{|x|} .
\end{aligned}
$$

This yields $f(t, 0)=\operatorname{arctg} t$. Thus, in order to obtain the existence result for equation (16) we will apply Theorem 2.

At the beginning let us notice that $F=\pi / 2$. In addition we have $k(t, s)=$ 
$\exp (-t-s)$ and $\varphi(x)=\sqrt{|x|}$. Thus assumption (v) is satisfied. Next, we have

$$
\begin{aligned}
\int_{0}^{t} k(t, s) d_{s} g(t, s) & =\int_{0}^{t} \exp (-t-s) \frac{t}{t+s} d s \\
& =\exp (-t) \int_{0}^{t} \frac{t}{t+s} \exp (-s) d s \\
& \leq \exp (-t) \int_{0}^{t} \exp (-s) d s \\
& =\exp (-t)(1-\exp (-t)) .
\end{aligned}
$$

This implies that the function $t \rightarrow \int_{0}^{t} k(t, s) d_{s} g(t, s)$ is bounded and tends to zero at infinity. Moreover, we have $K \leq 1 / 4$. Let us also notice that assumption (vi) is satisfied with the function $m(t)=t$. This yields

$$
\begin{aligned}
m(t) \int_{0}^{t} k(t, s) d_{s} g(t, s) & =t \int_{0}^{t} \frac{t \exp (-t) \exp (-s)}{t+s} d s \\
& \leq t(\exp (-t)-\exp (-2 t)) .
\end{aligned}
$$

Using standard methods of differential calculus we can show that the function $t \rightarrow t(\exp (-t)-\exp (-2 t))$ attains on the interval $\mathbf{R}_{+}$its maximum which can be estimated from above by the number 0.3 . This implies that $M \leq 0.3$. Notice also that $\|a\|=4 / e^{2}$.

Taking into account all facts established above we conclude that the inequality from (viii) has the form

$$
4 / e^{2}+\frac{\pi}{2} K \sqrt{r}+M r \sqrt{r} \leq r .
$$

Keeping in mind the above estimates of the constants $K$ and $M$ it is easily seen that the above inequality has a positive solution $r_{0}$. For example, $r_{0}=4$. Thus, in the light of Theorem 2 equation (16) has a solution in the space $B C\left(\mathrm{R}_{+}\right)$ which vanishes at infinity.

\section{REFERENCES}

1. Agarwal, R. P., O'Regan, D., and Wong, P. J. Y., Positive solutions of differential, difference and integral equations, Kluwer Academic Publishers, Dordrecht, 1999.

2. Argyros, I. K., Quadratic equations and applications to Chandrasekhar's and related equations, Bull. Austral. Math. Soc. 32 (1985), 275-282.

3. Argyros, I. K., On a class of quadratic integral equations with perturbations, Funct. Approx. Comment. Math. 20 (1992), 51-63.

4. Banaś, J., and Caballero Mena, J., Some properties of nonlinear Volterra-Stieltjes integral operators, Comput. Math. Appl. 49 (2005), 1565-1573. 
5. Banaś, J., and Dronka, J., Integral operators of Volterra-Stieltjes type, their properties and applications, Math. Comput. Modelling 32 (2000), 1321-1331.

6. Banaś, J., and Goebel, K., Measures of Noncompactness in Banach Spaces, Lecture Notes in Pure and Appl. Math. 60 (1980).

7. Banaś, J., Lecko, M., and El-Sayed, W. G., Existence theorems for some quadratic integral equations, J. Math. Anal. Appl. 222 (1998), 276-285.

8. Banaś, J., and O'Regan, D., Volterra-Stieltjes integral operators, Math. Comput. Modelling 41 (2005), 335-344.

9. Banaś, J., Rocha, J., and Sadarangani, K., Solvability of a nonlinear integral equation of Volterra type, J. Comput. Appl. Math. 157 (2003), 31-48.

10. Banaś, J., and Rzepka, B., On existence and asymptotic stability of solutions of a nonlinear integral equation, J. Math. Anal. Appl. 284 (2003), 165-173.

11. Banaś, J., and Sadarangani, K., Solvability of Volterra-Stieltjes operator-integral equations and their applications, Comput. Math. Appl. 41 (2001), 1535-1544.

12. Billingsley, P., Probability and Measure, Wiley, New York, 1979.

13. Burton, T. A., Volterra Integral and Differential Equations, Academic Press, New York, 1983.

14. Chandrasekhar, S., Radiative Transfer, Dover, New York, 1960.

15. Corduneanu, C., Integral Equations and Applications, Cambridge Univ. Press, Cambridge, 1991.

16. Darsow, W. F., Nguyeu, B., and Olsen, E. T., Copulas and Markov processes, Illinois J. Math. 36 (1992), 600-642.

17. Darwish, M. A., On quadratic integral equation of fractional orders, J. Math. Anal. Appl. (to appear).

18. Deimling, K., Nonlinear Functional Analysis, Springer Verlag, Berlin, 1985.

19. Dunford, N., and Schwartz, J. T., Linear Operators I, Int. Publ., Leyden, 1963.

20. Myškis, A. D., Linear differential equations with retarded argument, Nauka, Moscow, 1972 (in Russian).

21. Natanson, I. P., Theory of Functions of a Real Variable, Ungar, New York, 1960.

22. Sikorski, R., Real Functions, PWN, Warsaw, 1958 (in Polish).

23. Sklar, A., Fonctions de répartition á $n$ dimensions et leurs margens, Publ. Inst. Statist. Univ. Paris 8 (1959), 229-231.

24. Väth, M., Volterra and Integral Equations of Vector Functions, Pure and Applied Math., Marcel Dekker, New York, 2000.

25. Zabrejko, P. P., Koshelev, A. I., Krasnosel'skii, M. A., Mikhlin, S. G., Rakovschik, L. S., and Stetsenko, V. J., Integral Equations, Nordhoff, Leyden, 1975.

DEPARTMENT OF MATHEMATICS

RZESZÓW UNIVERSITY OF TECHNOLOGY

35-959 RZESZÓW, W. POLA 2

POLAND

E-mail: jbanas@prz.rzeszow.pl 